\title{
Visceral leishmaniasis in a patient taking adalimumab for rheumatoid arthritis
}

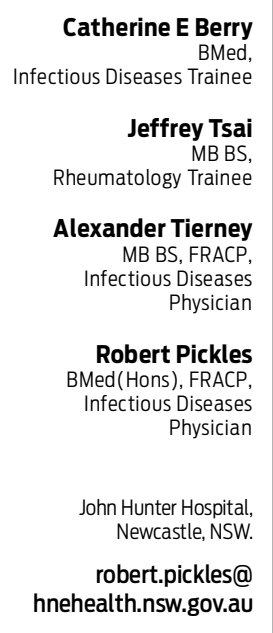

doi: 10.5694/mjal2.11003

\begin{abstract}
Opportunistic infections have been increasingly recognised with the advent of biological therapy for rheumatic disease. Visceral leishmaniasis $(\mathrm{VL})$ has been reported in Europe in association with tumour necrosis factor-alpha inhibitors. We report the first case of VL in an Australian returned traveller taking adalimumab.
\end{abstract}

\section{Clinical record}

A 69-year-old, Australian-born woman with a 14-year history of seropositive rheumatoid arthritis presented with a 3-week history of sweats and 1 week of severe malaise and postural dizziness. She had previously been treated with a variety of disease-modifying antirheumatic drugs (DMARDs) and was taking methotrexate $20 \mathrm{mg}$ weekly at the time of admission. She had suffered from an arthritic flare over the preceding 6 months and, as a result, was treated with adalimumab (an inhibitor of tumour necrosis factor-alpha [TNF- $\alpha$ ] and a type of biological DMARD) $40 \mathrm{mg}$ fortnightly for 8 weeks before her admission. The results of all pretreatment screening tests, including for latent tuberculosis, were negative. Her background history included atrial fibrillation, coronary artery disease, hypertension, pulmonary infiltrates that were presumed to be rheumatoid nodules (stable under computed tomography [CT] observation over a 3-year period). She was an exsmoker with a 40 pack-year history. Her travel history was extensive and, in particular, she had taken a 6-week trip to France, Portugal and Spain 5 months before presentation. She recalled some arthropod bites during 2 weeks in Andalusia, Spain.

On admission, her night sweats were found to represent intermittent fevers. She was hypotensive with a systolic blood pressure of $90 \mathrm{mmHg}$, and her spleen was palpable. Laboratory findings showed a C-reactive protein (CRP) level of $33.6 \mathrm{mg} / \mathrm{L}$ (reference interval [RI], 0-3.1 mg/L), erythrocyte sedimentation rate of $33 \mathrm{~mm} / \mathrm{h}(\mathrm{RI}, 0-10 \mathrm{~mm} / \mathrm{h}$ ), haemoglobin level of $117 \mathrm{~g} / \mathrm{L}$ (RI, 115-165 g/L) and albumin level of $31 \mathrm{~g} / \mathrm{L}$ (RI, 33-41 g/L). The results of multiple sets of blood cultures and an HIV test were negative.

The initial differential diagnosis included an opportunistic infection, malignancy or a medication effect. Methotrexate and adalimumab were no longer given.

Her clinical status deteriorated with progressively worsening malaise and fevers. Over the subsequent 3 weeks, she developed pancytopenia with a neutrophil count of $0.7 \times 10^{9} / \mathrm{L}\left(\mathrm{RI}, 1.8-7.7 \times 10^{9} / \mathrm{L}\right.$ ), haemoglobin level of $75 \mathrm{~g} / \mathrm{L}$ and platelet count of $32 \times 10^{9} / \mathrm{L}$ (RI, $150-400 \times 10^{9} / \mathrm{L}$ ). Total gammaglobulin concentration was $38.70 \mathrm{~g} / \mathrm{L}$ (RI, 6.45$13.9 \mathrm{~g} / \mathrm{L}$ ), and her ferritin level increased to $27401 \mu \mathrm{g} / \mathrm{L}$ (RI, $30-260 \mu \mathrm{g} / \mathrm{L}$ ). The results of an initial bone marrow biopsy did not assist diagnosis, although a trephine biopsy could not be performed.

A CT image of her chest showed unchanged pulmonary nodularity, and splenomegaly that was new since her previous CT chest scan. As there was still no diagnosis after 4 weeks of investigations, fine needle aspiration of a lung nodule was attempted, without success. Due to ongoing concerns about possible malignancy (pulmonary or haematological), a positron emission tomography scan was performed. The lung nodules were not fluorodeoxyglucose (FDG)-avid; however, the spleen was found to be intensely hypermetabolic, as shown by increased FDG uptake (Box 1).

Thrombocytopenia precluded a splenic biopsy, and a splenectomy was performed. The results of histopathological examination showed visceral leishmaniasis (VL) with multiple intracellular amastigotes within macrophages, while a repeat bone marrow biopsy also showed protozoa (Box 2). Polymerase chain reaction (PCR) testing of the splenic tissue was performed at St Vincent's Hospital, Sydney, and results were positive for Leishmania infantum. A retrospective review of the initial bone marrow biopsy found a single macrophage containing amastigotes.

The patient was treated with liposomal amphotericin on Days 1-5, 10, 17, 24, 31 and 38, with a total cumulative dose of $40 \mathrm{mg} / \mathrm{kg}$. By postoperative Day 30, her CRP level had decreased to $12.1 \mathrm{mg} / \mathrm{L}$, her haemoglobin level had improved to $132 \mathrm{~g} / \mathrm{L}$ and she had returned to her premorbid level of function. Her rheumatoid arthritis flared again 3 months later, and methotrexate therapy was recommenced. At 18 months, there has been no evidence of recurrent infection, and her rheumatoid arthritis has remained under moderate control; however, there is no current plan to recommence biological DMARD therapy.

\section{Discussion}

Kala-azar, meaning black fever in Hindi, is the visceral form of leishmaniasis. Worldwide, 500000 cases are reported per annum, and the disease is responsible for 60000 deaths annually. Unlike the often self-limiting cutaneous form, without treatment, VL is almost invariably fatal. ${ }^{1} \mathrm{VL}$ is principally caused by two of the 20 Leishmania protozoa known to cause disease in humans: L. infantum 


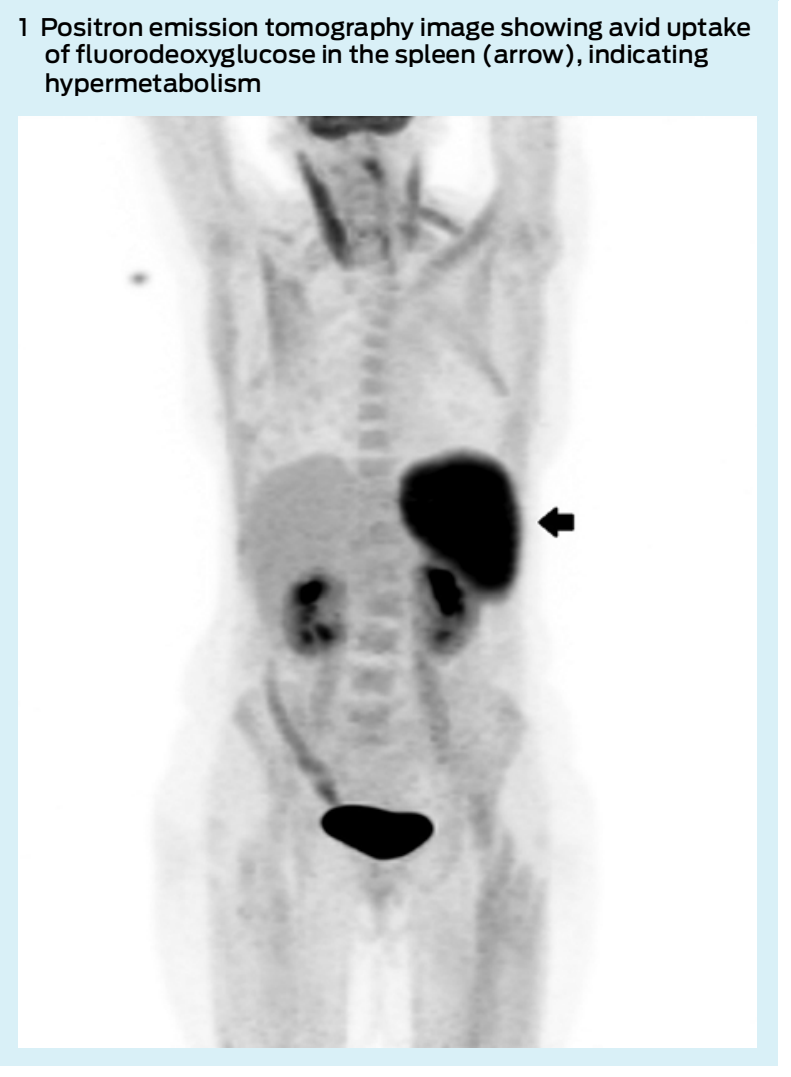

(also known as L. chagasi) and L. donovani. L. infantum, the species detected in the patient described here, tends to affect immunosuppressed patients and those at the extremes of age. In contrast, L. donovani can affect patients of any age.

Leishmaniasis is endemic to Africa, South America, Southern Europe and South Asia. The vectors are primarily species of the Phlebotomus sandfly; although in South America, the Lutzomyia longipalpis sandfly is responsible. Although leishmaniasis affecting humans has never been locally acquired in Australia, there does exist a species affecting kangaroos, believed to be spread by a dayfeeding midge, Forcipomyia (Lasiohelea) Kieffer. Depending on the species and environment, VL is variably a zoonosis or anthroponosis, and reservoirs include dogs, rodents and primates. ${ }^{1}$ Incubation times are derived from cases in returned travellers, including Australians. From these observations, it is clear that incubation can vary from 2 weeks to 18 months. $^{2}$ Further, travel to endemic areas for periods as short as 7 days has resulted in disease. ${ }^{3} \mathrm{VL}$ is significantly associated with HIV infection and other forms of immunosuppression.

This case brings to light a number of issues. First, the frequency of VL in association with biological DMARDs, particularly TNF- $\alpha$ inhibitors, optimal therapy for VL in this setting, and whether it is safe to recommence treatment with biological DMARDs after a diagnosis of VL. Second, what strategies could be considered to prevent VL in this population; and finally, whether diagnosis could have been expedited in our patient, thus avoiding splenectomy.

Leishmania are well adapted to survival and frequently cause latent infection. ${ }^{2}$ In an infection, the Leishmania promastigotes are taken up by phagocytes but also inhabit other cells, such as fibroblasts, and become amastigotes. They elicit a T-helper type 1 (Th1) response that produces interleukin-12 and recruits natural killer cells and CD8+ $\mathrm{T}$ cells. Production of interferon-gamma, activation of macrophages and release of $\mathrm{TNF}-\alpha$ promotes killing of parasites with nitric oxide. TNF- $\alpha$ also assists in proinflammatory signalling by producing a positive feedback loop, stimulating macrophages and promoting the Th1 response.

The association of VL with DMARDs emphasises the importance of TNF- $\alpha$ in maintaining immune control. Although VL is well described in association with nonbiological DMARDs such as methotrexate and corticosteroids, ${ }^{2}$ an increasing association with biological DMARDs has been recognised. We identified 15 reported cases of VL involving TNF- $\alpha$ inhibitors. ${ }^{2,4-10}$ With only one exception, patients were diagnosed in endemic areas of Southern Europe. The other patient was a British traveller returning from Malta. ${ }^{8}$ The underlying conditions varied but nine out of 15 were related to rheumatoid arthritis. Most were also taking other forms of immunosuppressive medications such as corticosteroids or other DMARDs. Most patients presented with the classic pentad of fever, cachexia, splenomegaly, hypergammaglobulinaemia and pancytopenia. Nevertheless, patients tended to have a protracted course between presentation and diagnosis. Diagnosis was most often made by isolating amastigotes from bone marrow; however, more than one bone marrow biopsy was frequently required for diagnosis. No other patient proceeded to splenectomy. One patient died and all others recovered. Twelve patients were treated with liposomal amphotericin, with total doses ranging from $18-50 \mathrm{mg} / \mathrm{kg}$. Treatment with $\mathrm{TNF}-\alpha$ inhibitors was recommenced in only three patients. ${ }^{6,9-10}$ Several patients recommenced non-biological DMARDs. There have been no reported cases of subsequent relapse of VL to date.

Pentavalent antimonials have been the mainstay of therapy for leishmaniasis, and these remain in wide use. However, difficulties with resistance, toxicity and quality control of generic forms of these medications limit their

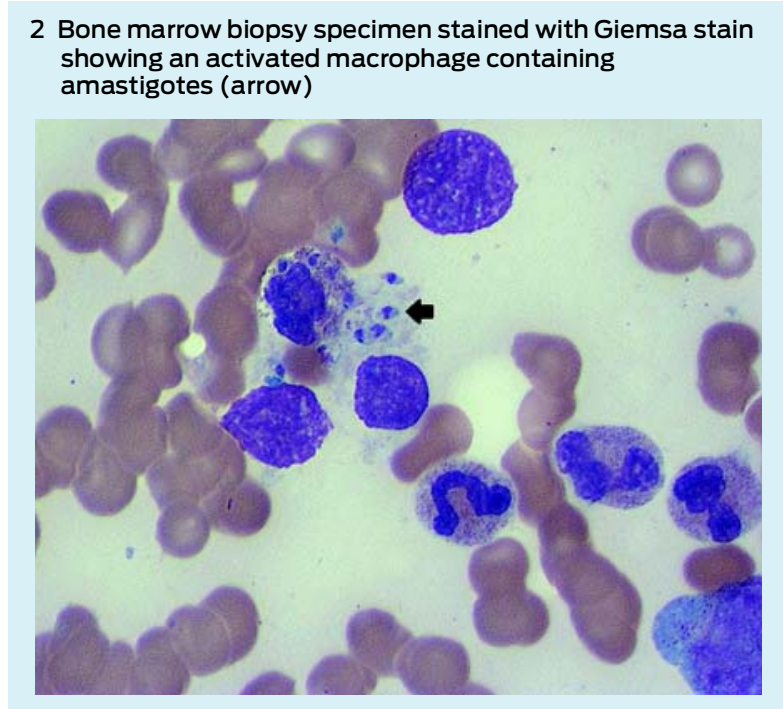


utility. ${ }^{1}$ Increasingly, the liposomal form of amphotericin is recognised as the least toxic treatment option and is currently the only agent approved by the Therapeutic Goods Administration for use in Australia. A variety of treatment protocols have been proposed, balancing cost and efficacy. Cure rates of $90 \%$ have been reported with doses as low as $12.5 \mathrm{mg} / \mathrm{kg}$ in India. ${ }^{11}$ However, with high relapse rates in the HIV-positive population, total doses of $20-60 \mathrm{mg} / \mathrm{kg}$ have been recommended. We chose a treatment protocol suitable for patients with HIV because we felt that our patient's degree of immunosuppression more closely reflected that of this population.

There are very few patients who have recommenced biological DMARD therapy after their episode of VL, so it is difficult to make a judgement about safety in this setting. Certainly there is a substantial theoretical risk, given the difficulty of achieving cure of the parasitic infection ${ }^{2}$ and the experience of relapse in patients with HIV infection. One option may be to monitor patients with Leishmania PCR testing, which is available in Australia and has been shown to accurately detect disease both acutely and in the context of relapse, and has been used in patients with and without HIV infection. ${ }^{1}$ This can be performed on both serum and tissue.

Several recent reports have discussed pretreatment screening of patients in endemic areas for VL, as is done for tuberculosis. ${ }^{2,4-5}$ This could be performed with either the leishmanin skin test (not available in Australia), a type of delayed hypersensitivity test, or serological testing. Generally, it is felt that the incidence is still too infrequent to justify such an intervention. Further, it is unclear whether prophylaxis or pretreatment eradication would be efficacious. At this stage, vector avoidance is the only strategy that can be recommended to patients.

Nevertheless, there is definite potential for future changes in the epidemiology of leishmaniasis. Increasing use of TNF- $\alpha$ inhibitors in endemic areas such as Brazil and the Indian subcontinent may put a much larger population at risk. Further, climate change may affect the geographical distribution of vectors and reservoirs, demanding continued vector surveillance beyond currently affected regions. ${ }^{1,6}$

A diagnosis for our patient would have been expedited by a higher index of suspicion of VL. VL poses a small but significant risk to immunosuppressed patients travelling to areas endemic for leishmaniasis and should be considered as a differential diagnosis in such patients presenting with pyrexia of unknown origin.

Competing interests: No relevant disclosures.

Received 24 Jun 2012, accepted 10 Dec 2012.

1 Magill AJ. Leishmania species: visceral (kala-azar), cutaneous, and mucosal leishmaniasis. In: Mandell GL, Bennett JE, Dolin R, editors. Mandell, Douglas, and Bennett's principles and practice of infectious diseases. Vol 2. Philadelphia: Churchill Livingstone/Elsevier, 2010: 3463-3480.

2 Bogdan C. Leishmaniasis in rheumatology, haematology and oncology: epidemiological, immunological and clinical aspects and caveats. Ann Rheum Dis 2012; 71 Suppl 2: i60-i66. doi: 10.1136/annrheumdis-2011-200596.

3 Hamilton A, Kelleher A, Marriott D. A case of severe visceral leishmaniasis resulting from travel to Greece. BMJ Case Rep 2009; 2009 pii: bcr06.2009.2036. doi:10.1136/bcr.06.2009.2036.

4 Khan A, Coakley A, Cosgrove C, Lockwood D. Let off the leash: kala-azar following the use of tumour necrosis factor antibodies. BMJ Case Rep 2010; 2010 pii: bcr0420102878. doi: 10.1136/bcr.04.2010.2878.

5 Cascio A, laria M, Iaria C. Leishmaniasis and biologic therapies for rheumatologic diseases. Semin Arthritis Rheum 2010; 40: e3-e5.

6 Erre GL, Mesina P, Tonelli N, Passiu G. Visceral leishmaniasis among immunosuppressed patients with rheumatic diseases. Clin Exp Rheumatol 2010; 28: 590-591.

7 Moltó A, Mateo L, Lloveras N, et al. Visceral leishmaniasis and macrophagic activation syndrome in a patient with rheumatoid arthritis under treatment with adalimumab. Joint Bone Spine 2010; 77: 271-273.

8 Kritikos K, Haritatos E, Tsigkos S, et al. An atypical presentation of visceral leishmaniasis infection in a patient with rheumatoid arthritis treated with infliximab. J Clin Rheumatol 2010; 16: 38-39.

9 De Leonardis F. Govoni M, Lo Monaco A, Trotta F. Visceral leishmaniasis and anti-TNF-alpha therapy: case report and review of the literature. Clin Exp Rheumatol 2009; 27: 503-506.

10 Fabre S, Gibert C, Lechiche C, et al. Visceral leishmaniasis infection in a rheumatoid arthritis patient treated with infliximab. Clin Exp Rheumatol 2005; 23: 891-892.

11 Sundar S, Chakravarty J, Agarwal D, et al. Single-dose liposomal amphotericin B for visceral leishmaniasis in India. NEngl J Med 2010; 362: 504-512. 